\title{
Orbital Solution and Physical Parameters of the Binary Cepheid AW Per
}

\author{
J. Vinko \\ JATE University, Szeged, and Konkoly Observatory, Budapest, Hungary
}

AW Per is a well-known binary Cepheid. Recently Welch \& Evans (1989) determined a spectroscopic orbit. Evans (1989) pointed out that the spectral type of the companion is incompatible with the mass function of the system. We re-determined the orbit of AW Per using both photometric and spectroscopic data. The result of the simultanuous least-squares fit can be seen on Fig.1. The orbital elements are very close to the results of Welch \& Evans. After correcting for the companion, the Cepheid's light and velocity curves were analyzed with the surface-brightness method. The details of the analysis will be published in MNRAS (Vinko, 1992). The mass of AW Per was found to be 6 solar masses, which confirms the mass-problem of the secondary. Observations are planned to continue at Konkoly Observatory.

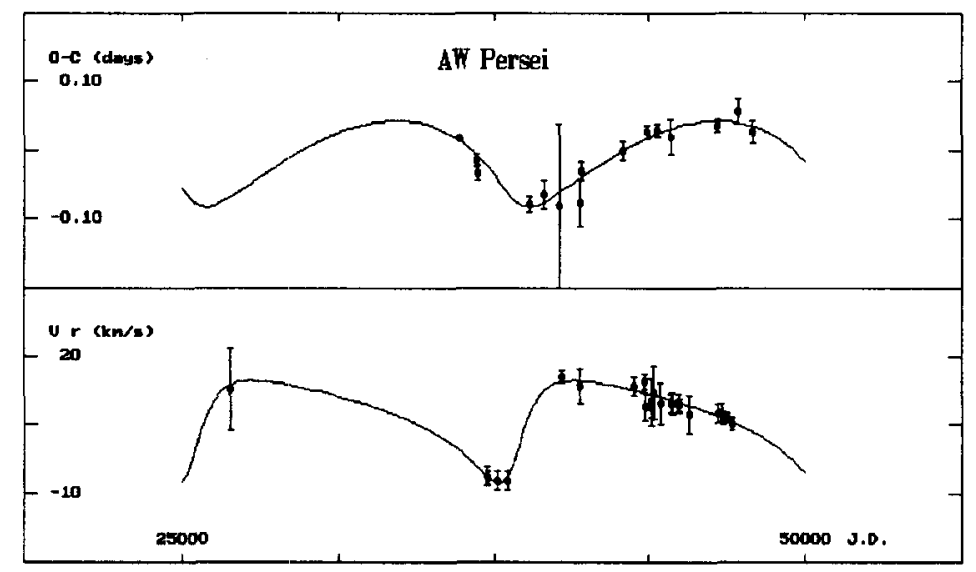

Figure 1. O-C and $\gamma$-velocity diagram of AW Per, assuming the results of the orbital solution.

\section{References:}

Evans,N.R., 1989, Astron.J. 97, 1737.

Vinko, J., 1992, Mon.Not.Royal Astron.Soc. (in press)

Welch,D.L. \& Evans,N.R., 1989, Astron.J. 97, 1153. 